Louisiana State University

LSU Digital Commons

Faculty Publications

Department of Physics \& Astronomy

1-1-1999

\title{
High harmonic generation at long wavelengths
}

\author{
B. Sheehy \\ Brookhaven National Laboratory \\ J. D.D. Martin \\ Brookhaven National Laboratory \\ L. F. Di Mauro \\ Brookhaven National Laboratory \\ P. Agostini \\ Service des Photons Atomes et Molécules \\ K. J. Schafer \\ Louisiana State University
}

See next page for additional authors

Follow this and additional works at: https://digitalcommons.Isu.edu/physics_astronomy_pubs

\section{Recommended Citation}

Sheehy, B., Martin, J., Di Mauro, L., Agostini, P., Schafer, K., Gaarde, M., \& Kulander, K. (1999). High harmonic generation at long wavelengths. Physical Review Letters, 83 (25), 5270-5273. https://doi.org/ 10.1103/PhysRevLett.83.5270

This Article is brought to you for free and open access by the Department of Physics \& Astronomy at LSU Digital Commons. It has been accepted for inclusion in Faculty Publications by an authorized administrator of LSU Digital Commons. For more information, please contact ir@lsu.edu. 


\section{Authors}

B. Sheehy, J. D.D. Martin, L. F. Di Mauro, P. Agostini, K. J. Schafer, M. B. Gaarde, and K. C. Kulander 


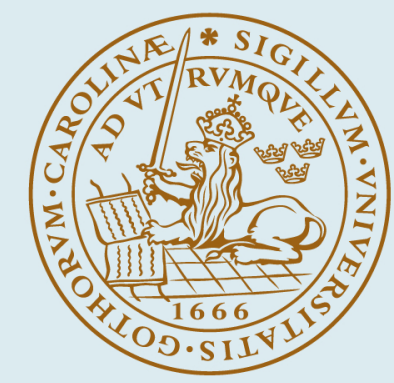

\section{LUND UNIVERSITY}

\section{High harmonic generation at long wavelengths}

Sheehy, B; Martin, J. D. D; DiMauro, L. F; Agostini, P; Schafer, Kenneth; Gaarde, Mette; Kulander, K. C

Published in:

Physical Review Letters

DOI:

10.1103/PhysRevLett.83.5270

1999

Link to publication

Citation for published version (APA):

Sheehy, B., Martin, J. D. D., DiMauro, L. F., Agostini, P., Schafer, K., Gaarde, M., \& Kulander, K. C. (1999). High harmonic generation at long wavelengths. Physical Review Letters, 83(25), 5270-5273.

https://doi.org/10.1103/PhysRevLett.83.5270

Total number of authors:

7

\section{General rights}

Unless other specific re-use rights are stated the following general rights apply:

Copyright and moral rights for the publications made accessible in the public portal are retained by the authors and/or other copyright owners and it is a condition of accessing publications that users recognise and abide by the legal requirements associated with these rights.

- Users may download and print one copy of any publication from the public portal for the purpose of private study or research.

- You may not further distribute the material or use it for any profit-making activity or commercial gain

- You may freely distribute the URL identifying the publication in the public portal

Read more about Creative commons licenses: https://creativecommons.org/licenses/

Take down policy

If you believe that this document breaches copyright please contact us providing details, and we will remove

access to the work immediately and investigate your claim. 


\title{
High Harmonic Generation at Long Wavelengths
}

\author{
B. Sheehy, ${ }^{1}$ J. D. D. Martin, ${ }^{1}$ L. F. DiMauro, ${ }^{1}$ P. Agostini, ${ }^{2}$ K. J. Schafer, ${ }^{3}$ M. B. Gaarde, ${ }^{3,4}$ and K. C. Kulander ${ }^{5}$ \\ ${ }^{1}$ Chemistry Department, Brookhaven National Laboratory, Upton, New York 11973 \\ ${ }^{2}$ SPAM, Centre d'Etudes de Saclay, 91191 Gif Sur Yvette, France \\ ${ }^{3}$ Department of Physics and Astronomy, Louisiana State University, Baton Rouge, Louisiana 70803-4001 \\ ${ }^{4}$ Department of Physics, Lund Institute of Technology, P.O. Box 118, S-22100 Lund, Sweden \\ ${ }^{5}$ TAMP, Lawrence Livermore National Laboratory, Livermore, California 94551
}

(Received 23 July 1999)

High harmonic radiation spectra up to 19th order in alkali metal vapors excited by an intense, picosecond mid-infrared $(3-4 \mu \mathrm{m})$ laser are reported and compared to theory. The strong-field dynamics in the alkali metal atoms exhibit significant differences from all previously studied systems due to the strong coupling between their ground and first excited states.

PACS numbers: $42.65 . \mathrm{Ky}, 31.90 .+\mathrm{s}, 32.80 . \mathrm{Fb}, 32.80 . \mathrm{Rm}$

High harmonic generation (HHG) resulting from intense laser-atom interactions is of particular interest because of its fundamental importance and practical applications. Most harmonic generation studies have been performed using pump lasers in the near-visible or ultraviolet. This has meant that multiphoton processes could be observed only in tightly bound systems such as the rare gases. HHG in rare gases provides coherent, short pulse, tabletop XUV sources, with spectra extending into the water window. These sources have found applications in atomic [1] and condensed matter [2] physics. They may also provide a route toward the generation of very short duration XUV pulses [3]. However, experimental progress has been limited, at least in part, by the lack of suitable materials for measuring VUV/XUV pulse lengths. For example, standard second harmonic intensity autocorrelation [4] requires frequency doubling materials which are not available at wavelengths below $400 \mathrm{~nm}$. To more readily understand the physics of the HHG process, especially the temporal properties, it may be possible to use intense mid-infrared (MIR) light to generate HHG in the UV/visible spectrum, where they can be characterized using conventional techniques.

In this Letter we report the first experimental and theoretical investigation of HHG by MIR (3-4 $\mu \mathrm{m})$ excitation in alkali metal vapors, specifically potassium and rubidium. Strong-field multiphoton processes, such as harmonic generation, using long wavelength pump lasers have until now been virtually unexplored. Harmonics extending to the 19th order of the fundamental field are observed.

The HHG process in alkali atoms using intense MIR radiation is also of fundamental interest as a strong-field phenomenon. For instance, the coupling between the ground and first excited state in MIR excitation of the alkali metals presents significant differences from the near-visible excitation of the rare gases. For an 11 photon ionization process in the two systems (alkali atoms and rare gases), the first excited state is separated from the ground state by 8-10 near-visible photons in the rare gases. The corresponding separation in the alkali metals is $4-5$ MIR pho- tons. This results in a strong coupling which can alter the ionization dynamics [5] and the high harmonic emission as compared to the rare gases.

Given the nature of HHG, we must be able to drive the laser-atom interaction into the strongly nonperturbative regime. The Keldysh parameter $\gamma=\sqrt{I_{p} / 2 U_{p}}$ [6], which compares a free electron's cycle averaged kinetic energy (the ponderomotive energy $U_{p}$ [7]) to the ionization potential $\left(I_{p}\right)$, provides a measure of the necessary strength of this interaction. As $\gamma$ becomes less than one, the ionization dynamics are dominated by tunneling and are well within the nonperturbative regime. Since the ponderomotive energy is wavelength and intensity dependent, the Keldysh parameter suggests the possibility of scaling the atomic interaction with intensity and wavelength. For instance, potassium atoms $\left(I_{p}=\right.$ $4.3 \mathrm{eV}$ ) interacting with a $1 \mathrm{TW} / \mathrm{cm}^{2}, 4 \mu \mathrm{m}$ field will have the same Keldysh parameter, implying similar ionization dynamics, as the well studied case of xenon with $70 \mathrm{TW} / \mathrm{cm}^{2}, 0.8 \mu \mathrm{m}$ pulses. The MIR source used in this experiment indeed provides ps pulses with peak intensities of the order of $1-2 \mathrm{TW} / \mathrm{cm}^{2}$.

Though $\gamma$ provides a guide for the scaling of the ionization, the scaling of the continuum dynamics is set by the ponderomotive energy $U_{p}$. HHG in the long wavelength, strong-field regime is well described in a semiclassical model as a two-step process which is initiated by tunneling ionization $[8,9]$. The wave packet generated in this process gains kinetic energy in the laser field and part of it returns to the ion core with a maximum kinetic energy of approximately $3 U_{p}$. Since $U_{p}$ scales quadratically with the wavelength, even for the same $\gamma$ the MIR field can in principle drive a higher order process than the corresponding visible field. The longer MIR cycle time, however, also implies a larger spreading of the continuum wave packet [8]. Consequently, scaling arguments based on any single parameter are only approximately predictive. Indeed, the results presented in this Letter show significant differences in the strong-field atomic dynamics as compared with all previous studies. 
Tunable MIR light is generated by difference frequency mixing the outputs of amplified titanium sapphire and $\mathrm{Nd}$ :YLF (yttrium lithium fluoride) mode-locked (ML) lasers. The two ML lasers are synchronized against a $80 \mathrm{MHz}$ reference rf-oscillator and regeneratively amplified at a one kilohertz repetition rate. Autocorrelation of the MIR idler beam yields a 1.9 ps pulse duration. Tuning of the titanium sapphire laser between $0.78-0.83 \mu \mathrm{m}$ produces a $100 \mu \mathrm{J}$ pulse energy over the wavelength range of 3-4 $\mu \mathrm{m}$ in a near-Gaussian mode.

The linear polarized MIR light is focused by an $\mathrm{f} / 4$ lens into a vacuum chamber near the exit aperture of an alkali metal oven. The effective length, $\mathcal{L}$, of the alkali metal $\cos ^{3} \theta$ density profile is $\simeq 1 \mathrm{~mm}$ full width at half maximum (FWHM). The oven temperature controls the vapor pressure in the range of $0.1-2$ torr. A 2 times diffraction limited beam waist of $40 \pm 5 \mu \mathrm{m}$ is measured using a scanning pinhole procedure. The confocal parameter, $b$, is $\sim 3 \mathrm{~mm}$. The high harmonics are 1:1 imaged onto the entrance slit of a $0.18 \mathrm{~m}$ flat-field air monochromator equipped with a gated, intensified charge-coupled device camera. The collection system's absolute efficiency at the third and fifth harmonic is determined by comparison to a calibrated photodiode. The full spectral response of the system is further refined using the emission spectrum of a Hg lamp [10]. The resulting uncertainty in the number of photons is $30 \%$ for the low harmonics and $50 \%$ for those higher than seventh order.

Figure 1 shows a typical composite low-resolution spectrum of rubidium atoms $\left(I_{p}=4.2 \mathrm{eV}\right)$ excited by a $1.9 \mathrm{ps}$, $3.6 \mu \mathrm{m}$ pulse. The distribution spans 9 orders of magnitude in photon counting and is not adjusted for the spectral response of the collection system. The spectrum is

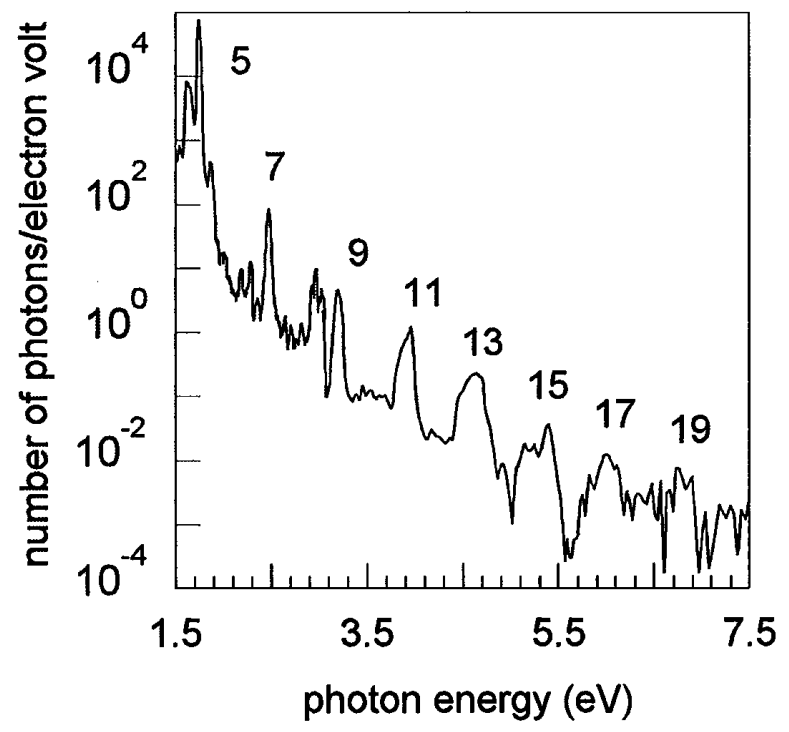

FIG. 1. High harmonic spectrum generated by excitation of rubidium atoms with $1.9 \mathrm{ps}, 3.6 \mu \mathrm{m}$ pulses. The additional lines in the spectrum can be assigned to fluorescence of $\mathrm{Rb}$. The spectrum is not corrected for the instrumental spectral response. recorded at a peak intensity of $2 \mathrm{TW} / \mathrm{cm}^{2}$. The measured saturation intensity is $1.1(4) \mathrm{TW} / \mathrm{cm}^{2}$ as determined by the intensity dependence of the total ion and harmonic yield. The spectrum shows a clear odd-harmonic series extending to the 19th order $(190 \mathrm{~nm})$ which is at the short wavelength limit of the optical detection system. The $3 U_{p}+I_{p}$ cutoff law [11] would give a maximum harmonic order of 23.

A few general features extracted from the experiment are as follows: (i) Depending on harmonic order, for the same number density, $\mathcal{N}$, and intensity, $\mathrm{Rb}$ is $3-6$ times more efficient than K. (ii) At these low densities, the harmonics show an $\mathcal{N}^{2}$ scaling which implies macroscopic coherence. (iii) The high harmonics show an intensitydependent scaling which is nonperturbative, i.e., less than an $\mathrm{I}^{q}$ power law, where $q$ is the harmonic order. (iv) Near field images verify that the emission is confined within a beam waist of the interaction volume. (v) The harmonic yield shows a weak dependence on the focal spot position relative to the alkali vapor distribution. These last two points illustrate that the harmonics originate from the intense region of a loosely focused beam $(b \simeq \mathcal{L})$ resulting in a optimized conversion geometry [12].

Figure 2(a) is a plot of the spectral linewidths as a function of harmonic order for $\mathrm{Rb}$ and $\mathrm{K}$ near the saturation
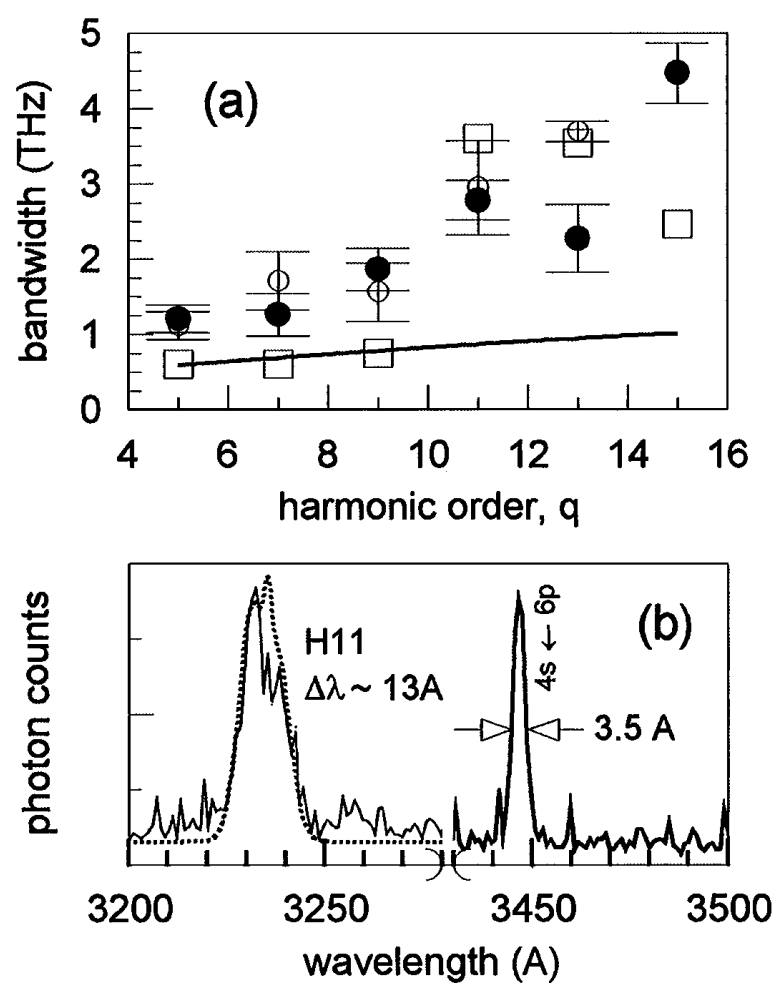

FIG. 2. (a) Linewidth as a function of harmonic order for $\mathrm{Rb}$ and $\mathrm{K}$. $\mathrm{K}(\circ)$ and $\mathrm{Rb}(\bullet)$ experimental linewidths are at 3.6 and $3.2 \mu \mathrm{m}$, respectively. The calculated $(\square) \mathrm{K}$ linewidths are for $3.6 \mu \mathrm{m}$ excitation. The solid line is calculated assuming a perturbative scaling. A typical measured (-) and calculated $(\cdots)$ harmonic line shape are plotted in (b). The $6 p \rightarrow 4 s \mathrm{~K}$ fluorescence provides an in situ calibration of the instrumental resolution. 
intensity. The filled $(\mathrm{Rb})$ and open $(\mathrm{K})$ circles are the measured FWHM linewidths for excitation at 3.2 and $3.6 \mu \mathrm{m}$, respectively. A high resolution spectrum of the 11th harmonic (H11) of $\mathrm{K}$ at $3.6 \mu \mathrm{m}$ is shown in Fig. 2(b). The H11 bandwidth has nearly 4 times the instrumental width and a complex line shape. In fact, the low order harmonics show a small increase in bandwidth followed by an abrupt increase near H11 which coincides with the binding energy. This trend is further exemplified by plotting the bandwidth increase expected for a perturbative $\sqrt{q}$ scaling [solid line in Fig. 2(a)] for a transform limited 1.9 ps fundamental pulse. Clearly, the higher harmonics show a strong deviation from the perturbative result and suggest, in principle, that significant temporal pulse compression is possible.

The harmonic results presented so far exhibit many similarities with the better-known near visible excitation of rare gas atoms, in both the general form of the spectrum and the large bandwidth of the higher order harmonics. This is a reflection of the universality of the continuum rescattering dynamics. However, as mentioned above, the alkali metals present several interesting and potentially useful contrasts to the rare gases. Chief among these is the strong coupling between the $n s$ ground state and the first excited $(n p)$ state. Figure 3 shows single-atom harmonic spectra calculated for a potassium atom in a $3.2 \mu \mathrm{m}, 80$ cycle laser pulse with a peak intensity of $1 \mathrm{TW} / \mathrm{cm}^{2}$. The atomic response is calculated by numerical integration of the timedependent Schrödinger equation using the single active electron (SAE) approximation [11] and the $\ell$-dependent pseudopotential of Stevens et al. [13], which we have modified slightly to give a better description of the $4 s \rightarrow n p$ dipole matrix elements.

Insight into the role of the lowest excited $(p)$ state can be obtained by comparing the full result (line in Fig. 3) with two approximate calculations. The first approximate dipole is calculated by projecting the full time-dependent

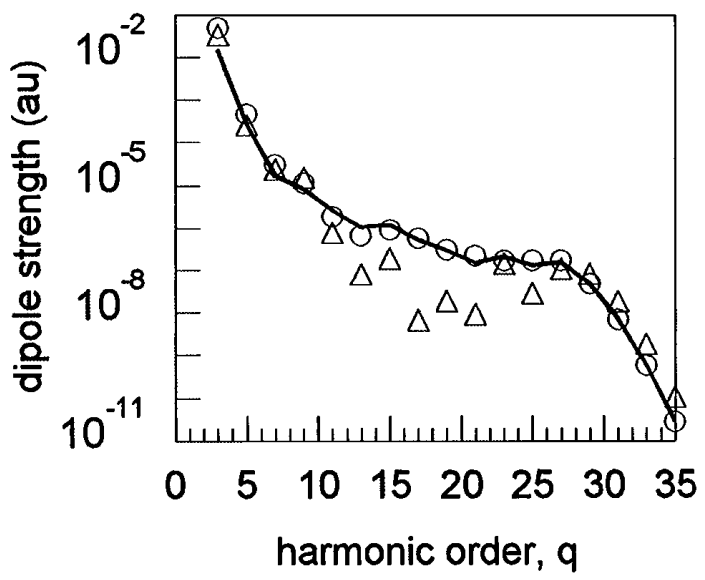

FIG. 3. Calculated $\mathrm{K}$ single atom harmonic spectrum for $3.2 \mu \mathrm{m}$ pulses. The full calculation (-) is compared to approximate dipoles calculated using the continuum wave packet plus the $4 s(\triangle)$ and $4 s+4 p(\bigcirc)$ only. wave function, $\psi(t)$, onto the field-free ground state at every time step. In potassium, where the ground state is the $4 s$, this approximate dipole is

$$
d(t)=\langle\psi(t)|z| \psi(t)\rangle \approx\langle\psi(t) \mid 4 s\rangle\langle 4 s|z| \psi(t)\rangle+\text { c.c. },
$$

where $z$ is the coordinate operator along the laser polarization direction. In essence, we assume that the field-dressed ground state is equivalent to the field-free ground state. This approximation (triangles in Fig. 3) works very well in the rare gases [11]. In potassium it exhibits significant deviations from the full result in both strength and cutoff energy. If the field-dressed ground state is assumed to be a linear combination of the ground and first excited state, then the approximate dipole is

$$
d(t) \approx\langle\psi(t)[|4 s\rangle\langle 4 s|+| 4 p\rangle\langle 4 p|] z \mid \psi(t)\rangle+\text { c.c. }
$$

The spectrum calculated in this manner (open circles in Fig. 3) agrees perfectly with the full calculation, illustrating the central role played by the first excited $n p$ state in HHG from alkali metals.

The experimental harmonics reflect the microscopic (atomic) and macroscopic (phase-matching and absorption) response of the nonlinear dipole. Therefore, we calculate the response of an ensemble of atoms in the nonlinear medium by solving the wave equation in the paraxial and slowly varying envelope approximations [14]. The full intensity dependent SAE atomic dipole moment discussed above is used as the source term of the nonlinear part of the polarization field [14].

Figures 2 and 4 display the calculated result for $\mathrm{K}$ and $\mathrm{Rb}$. The parameters used in the calculation are those determined from the experiment. The driving field is a $1.9 \mathrm{ps}$ (FWHM) $\mathrm{sech}^{2}$ pulse with a peak intensity of $1.2 \mathrm{TW} / \mathrm{cm}^{2}$. The spatial mode of the fundamental is modeled as a Gaussian with a $3.5 \mathrm{~mm}$ confocal parameter.

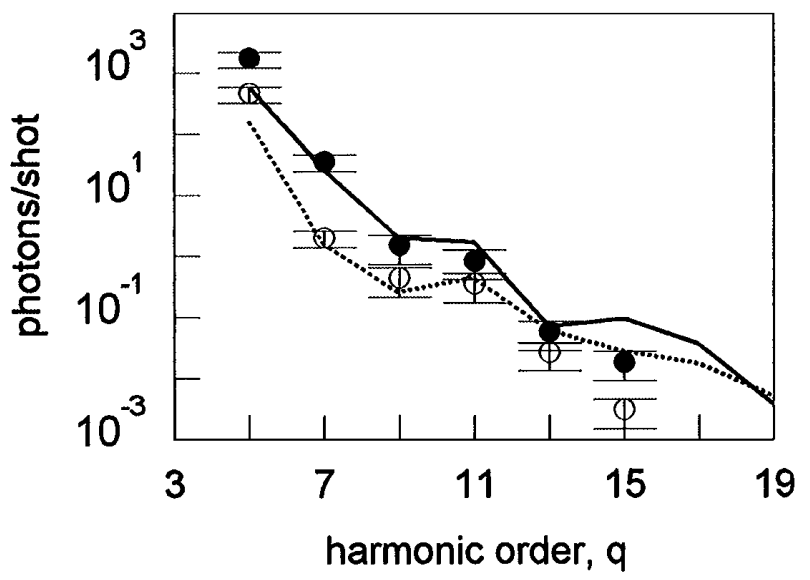

FIG. 4. Absolute number of photons as a function of harmonic order for $3.2 \mu \mathrm{m}$ excitation. The $\mathrm{K}\left({ }^{\circ}\right)$ and $\mathrm{Rb}(\bullet)$ experimental values are compared to the calculated $\mathrm{K}(\cdots)$ and $\mathrm{Rb}(-)$ distributions. The calculated yields are scaled by a factor of $10^{-3}$. 
As seen in Fig. 2(a), the K spectral widths observed in theory ( $\square$ ) and experiment (o) for a $3.6 \mu \mathrm{m}$ fundamental field are in good agreement. The widths of the lowestorder harmonics show a near perturbative $\sqrt{q}$ scaling harmonic order, whereas the bandwidth of the higher harmonics strongly deviates from this behavior. The calculated spectrum (dotted line) of the 11th harmonic is compared to the experimental result in Fig. 2(b). Our calculations indicate that the broad spectral features associated with the harmonics above the ionization threshold result from the intrinsic chirp that the harmonics possess due to the intensity dependence of the dipole phase [15].

In Fig. 4 we plot the measured (circles) and calculated (lines) number of photons for $\mathrm{K}$ and $\mathrm{Rb}$ at a pressure of 0.42 torr. The shape of the harmonic spectrum and the relative efficiency of the two atoms are well reproduced in the calculation. The $Z$-dependent efficiency is a single-atom effect, and is consistent with harmonic [12] and electron [16] distributions in rare gases. Although the qualitative behavior of the distribution of linewidths and harmonics for both atoms is good, a large discrepancy (roughly a factor of 1000) exists between the experimental and calculated absolute photon yields. Our calculations suggest imperfections in spatial mode of the focused MIR driving field can reduce the observed yield. Shot-averaged analysis does show a slight deviation from Gaussian of the MIR mode caused by the parametric process but not enough to completely explain this difference.

In summary, we have observe HHG from mid-infrared excitation of alkali vapors up to the 19th order. This investigation validates the Keldysh scaling of the laser-atom interaction at long wavelength and opens the possibility of studying nonperturbative strong-field interactions in a broad class of atomic systems with small binding energies. The coupling of the first excited $n p$ state is shown to play a major role in the HHG production, which provides an interesting and potentially useful contrast with the rare gas case. For example, it may be possible to use a second "coupling" laser as a means for achieving active control over the HHG process by altering either the $n s \rightarrow n p$ or the $n p \rightarrow$ continuum coupling. Furthermore, the realization of HHG spanning the visible/near-UV portion of the spectrum allows the application of frequency resolved optical gating [4] for complete characterization of the harmonic's amplitude and phase. This would probe both the potential for compression of single harmonics and the exciting possibility of synthesizing very short pulses by combining harmonics of different order [17].

The experiments were carried out at Brookhaven National Laboratory under Contract No. DE-AC0298CH10886 with the U.S. Department of Energy and supported by its Division of Chemical Sciences, Office of Basic Energy Sciences and BNL/LDRD No. 99-56. P. A. acknowledges travel support from NATO under Contract No. SA.5-2-05(RG910678). K. J. S. and M. B. G. acknowledge support from the Louisiana State Board of Regents through Grant No. LEQSF96-99-RD-A-14, and from the Swedish National Science Research Council. This work was also carried out in part under the auspices of the U.S. Department of Energy at the Lawrence Livermore National Laboratory under Contract No. W-7405-ENG-48.

[1] M. Gisselbrecht et al., Phys. Rev. Lett. 82, 4607 (1999).

[2] R. Haight, Appl. Opt. 35, 6445 (1996).

[3] S. E. Harris, J. J. Macklin, and T. W. Hänsch, Opt. Commun. 100, 487 (1993); G. Farkas and C. Toth, Phys. Lett. A 168, 447 (1992).

[4] R. Trebino et al., Rev. Sci. Instrum. 68, 3277 (1997).

[5] W. Nicklich et al., Phys. Rev. Lett. 69, 3455 (1992).

[6] L. V. Keldysh, Sov. Phys. JETP 20, 1307 (1965).

[7] $U_{p}$ measured in $\mathrm{eV}$ is given by $0.093 I \lambda^{2}$, where $I$ is the intensity in $\mathrm{TW} / \mathrm{cm}^{2}$ and $\lambda$ is the wavelength in $\mu \mathrm{m} . U_{p}$ is $1 \mathrm{eV}$ for $3.2 \mu \mathrm{m}$ excitation at $1 \mathrm{TW} / \mathrm{cm}^{2}$.

[8] K. J. Schafer et al., Phys. Rev. Lett. 70, 1599 (1993).

[9] P. B. Corkum, Phys. Rev. Lett. 71, 1994 (1993).

[10] J. Reader et al., Appl. Opt. 35, 78 (1996).

[11] K. C. Kulander, K. J. Schafer, and J. L. Krause, in Atoms in Intense Radiation Fields, edited by M. Gavrila (Academic Press, New York, 1992).

[12] A. L'Huillier et al., J. Nonlinear Opt. Phys. Mater. 4, 647 (1995).

[13] W. J. Stevens et al., Can. J. Chem. 70, 612 (1992).

[14] M. B. Gaarde et al., Phys. Rev. A 57, 4553 (1998).

[15] M. Lewenstein, P. Salières, and A. L'Huillier, Phys. Rev. A 52, 4747 (1995).

[16] B. Sheehy et al., Phys. Rev. A 58, 3942 (1998).

[17] Ph. Antoine, A. L'Huillier, and M. Lewenstein, Phys. Rev. Lett. 77, 1234 (1996). 